\title{
THE PERIODIC OPACITY OF WASSERMANN "ANTIGEN" IN PROGRESSIVELY INCREASING CONCENTRATIONS OF SODIUM CHLORIDE.**
}

\author{
By J. Holker, D.Sc., M.D. \\ From the Department of Pathology, University, Manchester.
}

(PLATE XXVI.)

THe author has recently shown $\left(1922^{1}\right)$ that, when a mixture of sermu and Wassermann "antigen" was treated in a certain way with progressively increasing concentrations of sodium chloride, ranging up to 10 per cent., it exhibited a periodic variation of opacity. He also showed that, when serum without antigen was similarly treated, the periodic opacity phenomenon was still more marked. When, however, Wassermann "antigen" without serum was similarly treated, the periodic opacity phenomenon was not exhibited. Instead there appeared a curve with a single extremely high maximum. Since carrying out the experiments cited, a considerable amount of work has been done with other colloids, and with concentrations of added electrolyte ranging up to saturation. In view of the wider experience thus gained, it became probable the Wassermann "antigen" also would exhibit the periodic phenomenon, if the concentration of sodium chloride was increased beyond 10 per cent. Accordingly, arrangements were made to test this conclusion by extending the experiments so as to include concentrations of added sodium chloricle ranging up to a saturated solution. The heart extract used was an acetoneinsoluble one prepared from a calf's heart by the method described by Bordet and Ruelens $\left(1919^{2}\right)$, and was cholesterinised by the addition thereto of an equal volume of a 1 per cent. solution of cholesterin in absolute alcohol.

The method of carrying out the series of experiments in the present investigation may be exemplified by describing in detail how a 1 in 100 suspension of antigen was treated with progressively increasing concentrations of sodium chloride. Into each of a series of resistance glass test-tubos was pipetted 0.5 c.e. of "antigen," and to this was then added rapilly 49.5 c.c. of a solution of sodium chloride which progressively increased in concentration in each successive test-tube. The resulting suspension was then placed in the thermostat at $40^{\circ} \mathrm{C}$. for four hours, and shaken initially and every hour afterwards. At the end of that time the opacity of the suspension was determined

* Received July $29,1922$. 
by means of the No. III. type of apparatus recently described by the author $\left(1921^{3}\right)$. The opacity of the suspension was then plotted

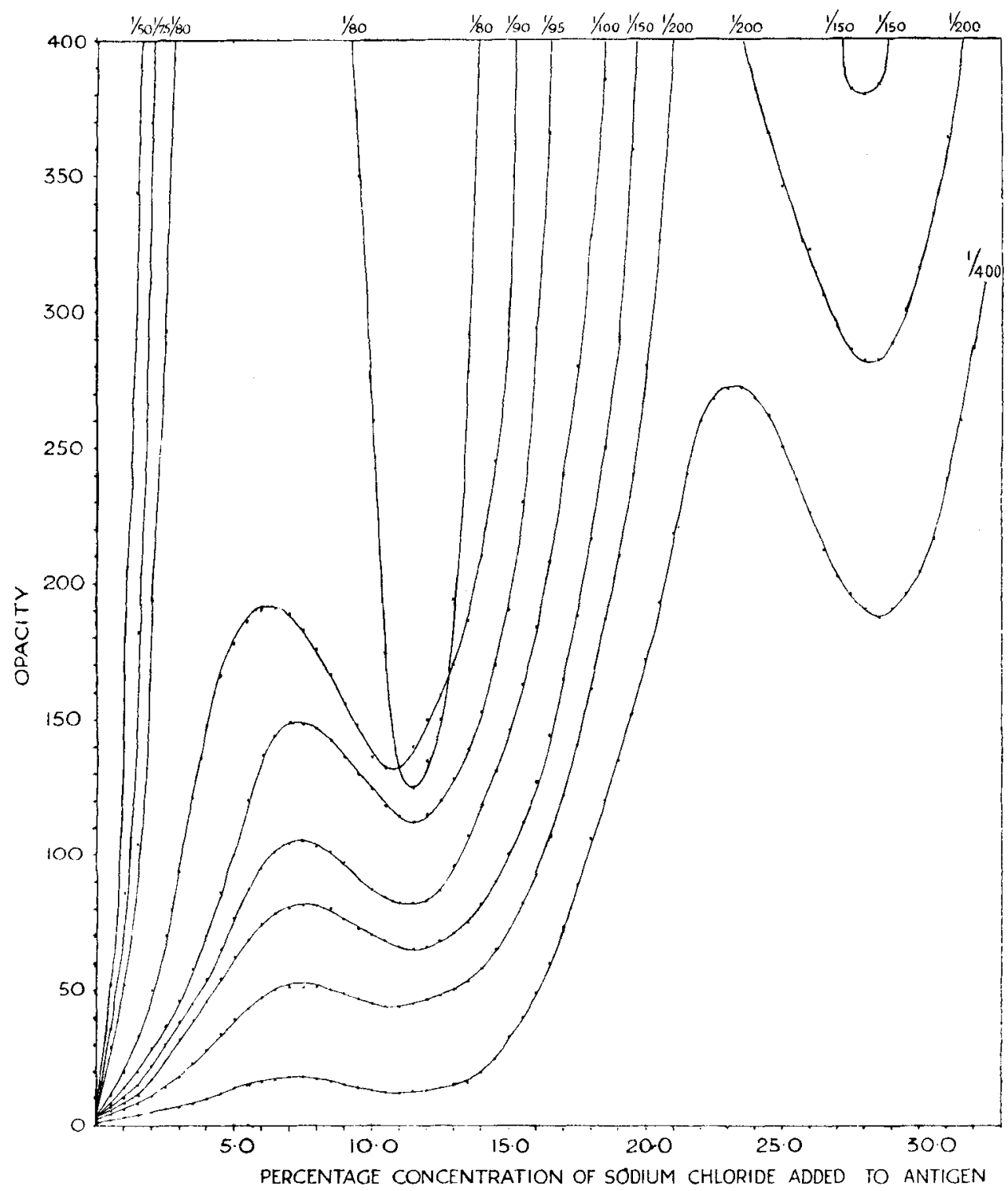

Fig. 1.-The Periodic Opacity of various Dilutions of Wassermann " Antigen " in progressively increasing concentrations of Sodium Chloride.

(The Dilutions of the "Antigen" are marked at the tops of the curves.)

against the concentration of the sodium chloride. Other concentrations of antigen were similarly treated with sodium chloride, and gradually in this way there was built up the chart of results shown in Fig. 1. 
A glance at the chart (see Fig. 1) shows at once that the Wassermann "antigen" exhibits a definite periodicity in its reaction to sodium chloride. The curves are, however, strikingly different from those for serum alone and serum plus antigen. Within the range limited by 10 per cent. sodium chloride, antigen alone had one oscillation only, whereas antigen plus serum had three, and serum alone had four. Evidently then, antigen had a markedly depressant action on the periodicity of serum. With antigen the initial part of the curves was a long lead. With serum the initial part of the curve was a long lag. With antigen the oscillations were only two in number. With serum diluted five times (see Fig. 2) the oscillations were eleven in number. With antigen the amplitude of the oscillations was so great that even with dilutions of 1 in 100 the periodic opacity

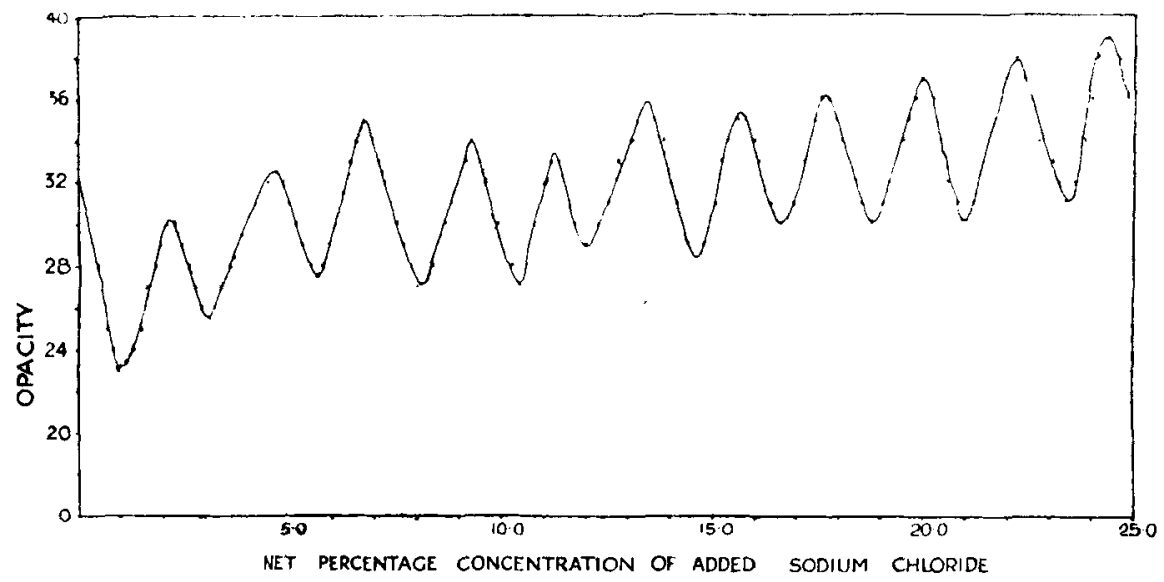

Frg. 2.-The Periodic Opacity of "Inactivated" Human Serum diluted five times with progressively increasing concentrations of Sodium Chloride.

phenomenon was visible to the naked eye, and capable of being photographed. One such photograph of antigen diluted eighty-five times is shown in Plate XXVI. Moreover, for those curves representing suspensions stronger than about 1 in 100 of antigen, the physical condition of the suspension at the maxima was visibly different from that at the minima. At the maxima flocculation had set in, and, on allowing the tubes to stand, the suspension cleared. On the other hand at the minima the suspension remained turbid. On shaking the suspension, provided, however, that it had not been allowed to stand for too long a time, the tubes at or near the maxima were seen to be very much more turbid than those at or near the minima. If the tubes stood for too long a time, it was difficult to separate the aggregated particles by mere shaking. For those curves representing suspensions weaker than about 1 in 100 of antigen, the periodic opacity was not readily visible, and finally became impossible to detect with the naked eye. With the aid of the opacimeter, however, the 
phenomenon was still olsserved in dilutions of 1 in 400 , and it was of interest to observe that the position of the maxima and minima in these curves agreed with those for curves representing suspension of 1 in 100 or stronger. With serum the amplitude of the oscillations was so small that it was impossible to detect any periodicity on looking at the test-tubes with the naked eye, and it was only when the opacimeter was used that the phenomenon was observed. Again, with antigen the amplitude of the second of the two oscillations was markedly greater than that of the first. With serum the eleven oscillations approximated fairly closely to an average.

A convenient method of measuring the periodicity of these curves has been found to be the ratio between the amplitude and the length of the period. A complete oscillation was taken to be that part of the curve lying between two successive minima. The amplitude of the oscillation was expressed in degrees of opacity and was taken to be the length of the pexpendicular dropped from the maximum to the line drawn through the two minima. The length of the oscillation was expressed as the difference in the normality of the net amount of added sodium chloride at the minima. The ratio of the amplitude to the length of the period was termed the characterising ratio of the period. For the serum-NaCl complex, the average characterising ratio for the eleven oscillations was 16.5. For the antigen- $\mathrm{NaCl}$ complex the characterising ratios were as follows :-

\begin{tabular}{|c|c|c|c|c|}
\hline \multirow[b]{2}{*}{ Dilution of Antigen. } & & & \multicolumn{2}{|c|}{ Characterising Ratio. } \\
\hline & & & 1st Period. & 2nd Period. \\
\hline 1 in 400 & - & . & . $5 \cdot 31$ & $32 \cdot 6$ \\
\hline 1 in 200 & • & . & . $10 \cdot 9$ & $\left\{\begin{array}{l}\text { maximum opacity } \\
\text { too high to be } \\
\text { measured }\end{array}\right.$ \\
\hline 1 in 150 & - & - & $15 \cdot 2$ & ", \\
\hline 1 in 100 & . & • & $21 \cdot 9$ &, \\
\hline 1 in 95 & , & . & . $29 \cdot 5$ & ", \\
\hline 1 in 90 & & . & - $\quad 38.9$ & , \\
\hline 1 in 80 & - & & $\cdot\{m$ & $\begin{array}{l}\text { opacity too high to } \\
\text { e measured }\end{array}$ \\
\hline
\end{tabular}

From these figures it was seen that the characterising ratio of antigen for the second period was much greater than that for the first. With serum the characterising ratio of each period closely approximated to the average. With serum plus antigen, however, the second characterising ratio was less than the first, and the third less than the second. The fact that the mixture of "antigen" (which has an increasing degree of periodicity) with serum (which has a uniform degree of periodicity), yielded a curve with a diminishing degree of periodicity, suggested that a definite combination had taken place between the antigen and serum with the production of a new complex whose properties were different from those of either of its constituents.

With regard to the application of these results it must be remembered that the Wassermann reaction is an extremely complex JOURx. OF PATII.- VOL. Xxv. 
one, involving the interaction of five different colloids, and that, hitherto, there have been available very few quantitative data of a physicochemical nature which would be suitable for the study of its mechanism. Indeed, until very recently, there have been no useful data available concerning the profound reaction between even the simple substances sodium chloride and the Wassermann reagents. In the author's opinion before much progress can be made in elucidating the mechanism of the Wassermann and other serum reactions, a wide field will have to be explored, which will cover not only the reactions of pathology in particular, but also those of colloid chemistry in general. It is to be remembered also, that in the Wassermann reaction the concentration of the sodium chloride used is limited to 0.85 per cent., in order to maintain the correct osmotic pressure for the suspension of red corpuscles. But in determining the best conditions for the colloidal interaction of serum and "antigen" we are not limited to physiologically normal saline, but can choose that concentration of sodium chloride which will give the best practical differentiation between negative and positive sera. It seems even probable that for practical purposes the best differentiation will be obtained with a saline which is not isotonic to rod corpuscles.

The expenses of this research have been defrayed partly by a grant from the Department of Pathology, for which I thank Professor H. R. Dean, and partly by a grant from the Medical Research Council, to whom an acknowledgment is also due.

\section{REFERENCES.}

1. Holker, J. . . . . . . "The Opacity of a Mixture of Serum and Wassermann 'Antigen' in progressively increasing Concentrations of Sodium Chloride," Journ. Path. and Bacteriol., 1922, xxv. 291.

2. Bordet, J., AND Ruelens, L'antigène syphilitique de l'Institut Pasteur de G. Bruxelles, Compt. rend. de la Soc. de Biol, 1919, lxxxii. 880.

3. HoLker, J. . . . . Methods of Measuring the Opacity of Liquids," Biochem. Journ., 1921, xv. 216.

\section{DESCRIPTION OF PLATE XXVI.}

Wassermann "antigen" diluted eighty-five times with progressively increasing conceritrations of sodium chloride. 


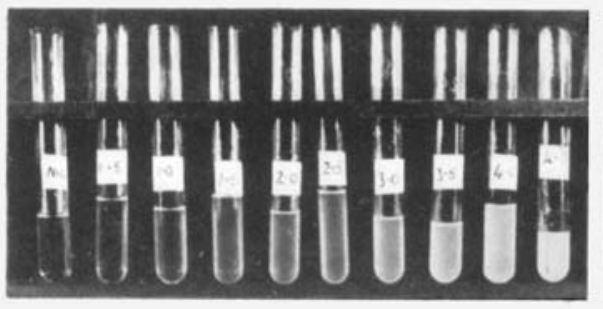

I

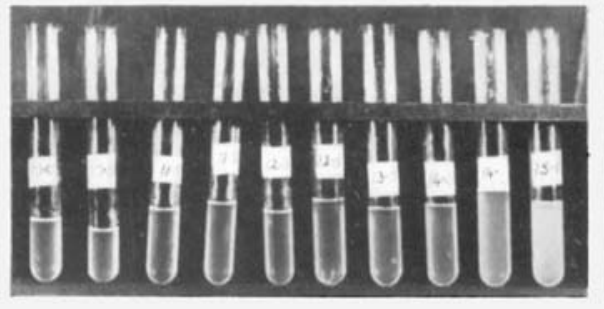

3

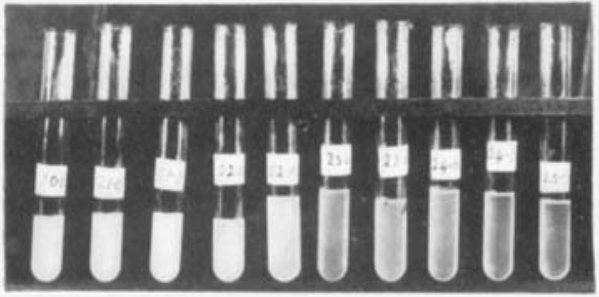

5

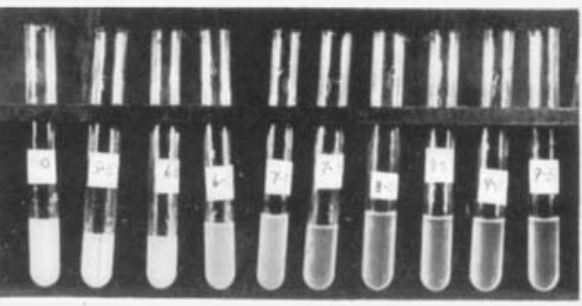

2

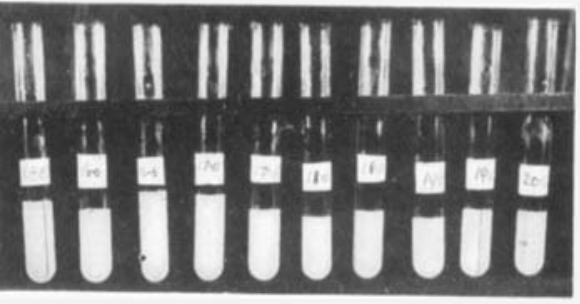

4

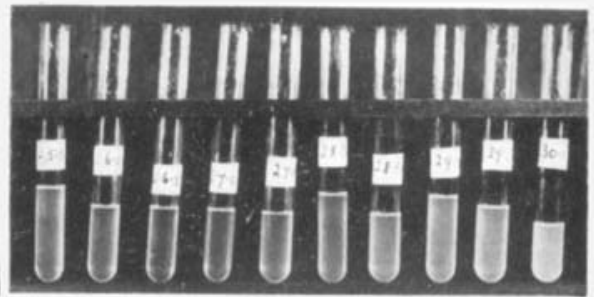

6

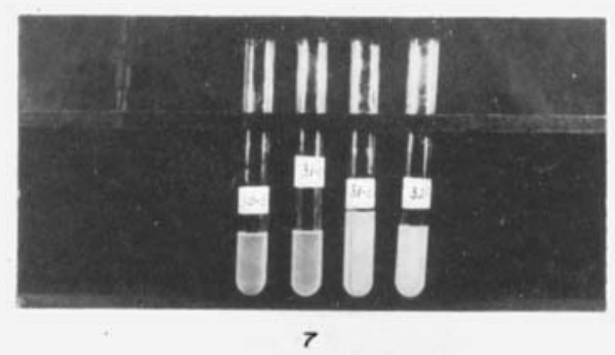

